\title{
BLOCKCHAIN APPLICATION FOR CONTRACT SCHEMES IN THE CONSTRUCTION INDUSTRY
}

\author{
GIULIA PATTINI ${ }^{1}$, GIUSEPPE MARTINO DI GIUDA ${ }^{1}$, and LAVINIA CHIARA \\ TAGLIABUE $^{2}$ \\ ${ }^{1}$ Dept of Built Environment and Construction Engineering, Politecnico di Milano, Milan, Italy \\ ${ }^{2}$ Dept of Civil, Environmental, Architectural Engineering and Mathematics, University of \\ Brescia, Brescia, Italy
}

\begin{abstract}
The proposed research aims at illustrating how Blockchain technology can support the contract execution optimizing and assuring a transparent information flow during the phases of the construction process. The traditional approach, chased in the industry, is indeed more hierarchical than networked, resulting in a high fragmentation of contracts and the break of companies in numerous tiers. This context prevents effective collaboration, hindering the achievement of the project objectives. The recent digital transition, guided by Building Information Modeling (BIM), has promised the creation of a shared environment for the information created and exchanged during the entire process, in favor of collaboration and reduction of critical issues typical related to the sector. Despite the initial promises, the use of BIM has shown problems related to trust and transparency of information, not encouraging participants to collaborate in meeting common goals. For these reasons, the study aims to investigate the potential of Blockchain technology in the management of the information flow to ensure transparency and stability of the process. The research proposes four frameworks showing how Blockchain can be integrated with the construction contract to support the execution of each phase, highlighting the improvement of information sharing, traceability of each activity or service and support for collaboration.
\end{abstract}

Keywords: Information flow, Construction contract, Smart contracts, Information trust, Distributed ledger technology, Lean process, Collaborative process, Construction automation.

\section{INTRODUCTION}

Compared to other sectors, the construction industry trend is accused of low productivity and poor performance mainly due to its unique characteristics (McKinsey Global Institute 2017). The hierarchical approach, the fragmentation of processes and the inability to maintain trustable longterm relationship are the factors that most influence the sector development (European Construction Sector Observatory 2018). Such an environment negatively influences relations among the parties who, finding themselves in an uncertain and fragmented context, have no incentive to share their information and work together to achieve common goals (Gálvez-Martos et al. 2018). This situation is characterized by the presence of information asymmetry that, driving opportunistic behaviors among the participants, affects the outputs of the construction process. The parties involved, not having the trust in the information exchanged (Hunhevicz and Hall 2019), prefer to carry out their tasks aiming at a personal profit to the detriment of the project objectives. Intending 
to distance themselves from the traditional and unfavorable dynamics described above, a few operators of the sector have proposed transformations of procedures and implementations of best practices aimed at overcoming the negative factors and redefining positively the traditional patterns of the industry. As the construction sector has the lowest digitalization index compared to others (Tezel et al. 2019), the introduction of new technologies is identified as one of the main changes to be pursued in order transform and improve the traditional approach to the process.

\section{BIM AS THE DRIVER OF DIGITAL TRANSITION}

The digital transition appears to be the leading impulse for change (European Commission 2019) and, in particular, the advent of Building Information Modeling in the sector supports transparent project development, fostering collaboration and fair information sharing among participants (Li et al. 2019). At the bottom of BIM, there is a Common Data Environment that contains and stores all project information in a comprehensible and semantic way, reducing the typical errors due to inconsistencies and disconnections among documents (Turk and Klinc 2017). The pursuit of a BIM-based process is therefore in line with collaborative nature of the industry and allows to significantly improve the relationships among the parties involved by supporting the digital visualization of the project, phase planning and communication among parties (Ye et al. 2018).

Despite the promise to eliminate the traditional information asymmetry that hinders transparent data sharing and honest collaboration among participants, the gradual adoption of BIM process has shown difficulties in management of information flow and, in particular, about the reliability, sharing, traceability and ownership of the information produced and exchanged during the implementation of the process. In a long term period, the presence of such issues and ambiguities discourages people from collaborating and sharing information truthfully, disturbing the process performance (Dounas et al. 2019). For these reasons, the research proposes the introduction of Blockchain technology which, guarantees the reliability of information (Nawari and Ravindran 2019). Although Blockchain is not yet actually adopted in the construction industry, the research illustrates its potential to support a collaborative environment and to manage a trusty, traceable and automated contract execution (Mathews et al. 2017).

\section{BLOCKCHAIN IMPLEMENTATION IN THE CONSTRUCTION INDUSTRY}

Thanks to its ability to revolutionize multiple industries (Osservatorio Blockchain and Distributed Ledger 2020), it is possible to consider Blockchain as the main technology qualifying the current digital transition. It belongs to Distributed Ledger Technologies (DLTs) and it is defined as trustless because it guarantees a decentralized system that preserves information ensuring trust, immutability and truth without the control of a third authority (Lai and Lee Kuo Chuen 2017), which means that it minimizes the amount of trust required from any single actor in the system by distributing trust among different actors in the system incentivizing actors to cooperate with defined rules. Its structure is divided into three layers and permits to understand the technology operation. The first layer is about the technological infrastructure needed for its functioning, the second defines the type of the ledger, its structure and accessibility, and rules for recording data on the ledger, and the third represents its applications, such as Smart Contracts (San et al. 2019).

Smart Contracts, based on Blockchain technology, are computational contracts composed of computer codes that, upon the occurrence of a specific prearranged condition, are able to execute themselves automatically following predetermined functions. The adoption of computational contracts revolutionizes the traditional contract increasing the efficiency of the process and reduces the number of transactions and legal costs (Hsiao 2017). The distributed nature of the Blockchain is the first relevant aspect for the implementation of Smart Contracts as it facilitates relations 
between participants and eliminates the need to control the fulfilment of a given contractual clause. Moreover, given the programmable nature of the Blockchain, it is possible to implement selfexecuting and self-enforcing contracts that allow the direct involvement of the contracting parties, thus overcoming the civil law system based on a third authority that verifies, certifies and implements the contractual terms.

Although the disruptive nature of Blockchain and Smart Contracts may offer potential benefits to the construction process, the novelty of the technology gives rise to debates on its actual applicability and usefulness. The main obstacles to implementation are identified in (i) the change in human mindset, (ii) the presence of unforeseen events not definable ex-ante and (iii) the definition of legislation dedicated to its adoption (Rodrigues et al. 2018). However, since collaboration and competition in any industrial process are fair when information is shared equally, the Blockchain adoption in the construction industry could result in an interesting research field. Due to the project requirements complexity and the variety of disciplines, a simple traditional contract isn't adequate to guarantee and support collaboration among parties. In addition, the current implementation of the BIM process is characterized by issues related to ownership, traceability and reliability of the information contained in the model. Faced with these problems, the idea of combining Blockchain technology with BIM is advantageous. This integration supports the development of a Common Data Environment no longer centralized but distributed among parties and the shift from a traditional contract scheme to a collaborative and digital one (Koutsogiannis and Berntsen 2017).

\section{BLOCKCHAIN-BASED BIM PROCESS FOR THE CONTRACT EXECUTION}

Although the stipulation of a construction contract regulates the roles and relations between the parties and the recent digital transition supports information flow improvement, the strong resistance to change, the slow adoption of new technology and the regulatory gap hinder the development of a collaborative agreement and a transparent information flow.

The idea of developing a Blockchain-based BIM process, therefore, ensures that every single change to the model is accurately recorded, thereby increasing the reliability, traceability and ownership of the information it contains and improving collaboration and trust between the parties involved (ARUP 2019). The advent of BIM has supported a data-driven approach influencing the use of information, holding up the shift from unstructured information to structured information. The advent of the Blockchain will lead to the transformation of contractual clauses, from expressions in descriptive language to expressions in computational language, abandoning the opportunistic behavior due to a free interpretation of the clauses in a traditional contract. In the legal environment typical of the construction sector, the possibility of making certain processes automated is a valid area of experimentation. Also, the implementation of Smart Contracts, based on the distributed Blockchain environment, fits with the nature of multi-party contractual agreements created to regulate and support the use of BIM. After the description of the achievable benefits through the development of a Blockchain-based BIM process, the following four frameworks (Figure 1) explore the potential applications of the distributed ledger and the Smart Contracts in any phase of the process, highlighting the potential of these tools in optimizing and supporting the traditional execution of the construction contracts.

\subsection{Design Phase}

The distributed ledger shared among parties supports planning and design phases, allowing a better understanding of the project information and those responsible for each activity. The project requirements and guidelines defined by the client, as well as all design packages to be completed, 
are registered on the Blockchain and therefore identified by all the contracting parties. In addition to a transparent and reliable sharing of information to support the development of the design phase, programming and use of Smart Contracts is enabled by the adoption of Blockchain.

In this first phase, Smart Contracts can be used to manage payments to design teams, with the automatic release of payments at the end of each project verification cycle. The BIM model is drawn up based on the client's guidelines, the compliance of each design level is verified through code and model checking software. On the basis of the verification results obtained, the Smart Contracts are automatically performed by releasing the corresponding fee.

\subsection{Tender Phase}

During the tender phase, the Blockchain ledger is useful for both the client and the participants. The first party deposits all the tender documents and evaluation criteria in an immutable manner, guaranteeing participants maximum fairness in the sharing of information and the same possibility to access all projects parts. In the same way, participants can deposit the tender offers on the platform, which become immutable and transparent to all.

The bids evaluation and comparison can be carried out using Smart Contracts programmed on the basis of the evaluation criteria defined by the client. The results of the tender evaluation are compared in a transparent way, ensuring an unambiguous selection of the winner.

\subsection{Construction Phase}

During the execution phase of the work, the platform can be used both to monitor the work progress and to control the development of the supply chain. BIM model relating to the work can be updated at the same time as the progress of the work, thus tracing the evolution of the activities envisaged in the work program and the parties responsible for each one. Similarly, information relating to the materials and products delivery to the construction site can be recorded on Blockchain, guaranteeing full transparency on the materials used.

The Smart Contracts can be programmed both on the basis of the milestones programmed and on the deadlines of the deliveries. On completion of each task and on delivery of each order, the information stored on the Blockchain allows the Smart Contracts to be automatically started and the payments to the authors of the task are released. This context, ensuring payments, provides an incentive for work teams to complete each task in compliance with the contractual terms.

\subsection{Maintenance Phase}

All information related to the maintenance activities of the building systems can be recorded on the Blockchain. The collection of information related to the operation and maintenance of the equipment makes it possible to monitor the regularity of the services provided by the contractors. Smart Contracts can be programmed based on the periodic maintenance activities to be carried out. Upon completion of each planned activity, the data is deposited and updated by the work teams in the model and the Smart Contracts automatically provide the payments.

The efficiency of these technologies increases in Smart Buildings in which the plants are able to autonomously detect the performance of regular maintenance and services according to the established tasks. In this way, the information related to the activity is automatically associated with the real object, existing in the model, and then recorded on the Blockchain. This information is the input for Smart Contracts execution, which automatically provides payment. 

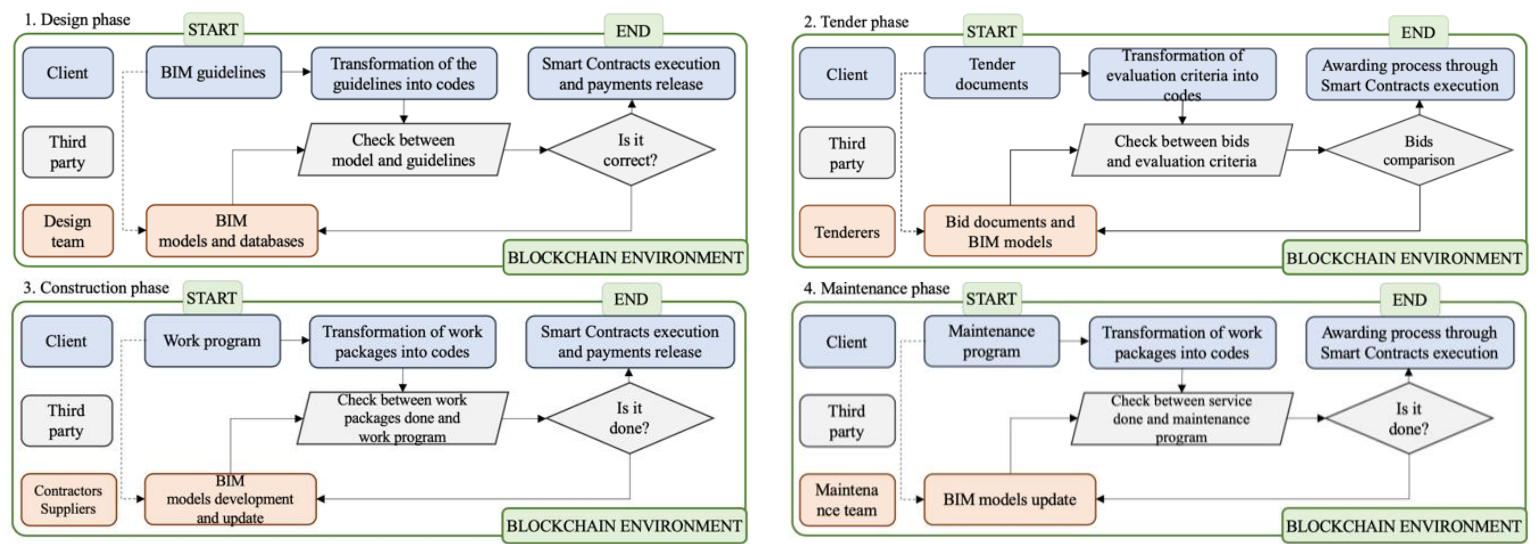

Figure 1. Construction process execution through Blockchain-based BIM process.

\section{CONCLUSIONS AND FURTHER DEVELOPMENTS}

The research carried out illustrates how Blockchain technology could be integrated and adopted in the construction process, highlighting the main benefits offered with regard to the rooted problem of the information trust. Indeed, the distributed digital environment offered by Blockchain eliminates the problems of information asymmetry by ensuring trust and transparency of data shared throughout the process. The presence of a distributed ledger eliminates the centralization of ownership in a single entity and reduces misuse and corruption of information by making the data a "legal proof" (Erri Pradeep et al. 2019). In this way, the process development (i.e. the digital models and the real work) with the support of the Blockchain and the Smart Contracts execution makes it possible to reduce the misunderstanding and delays often due to discrepancies and nonconformities between project drawings or models during various phases.

The main weaknesses of the BIM process such as the information trust, ownership and traceability and the effective integration of all the parties can be supported through the Blockchain adoption. Although due to the dynamic nature of the industry, it is unlikely to make the entire contract execution process automatic, the only clauses that can be defined ex-ante with certainty or that can be translated into computational language can be considered in the Smart Contracts. In this way, the contractual scheme used is characterized by both traditional contracts, considered as the main reference resource, and the integration of some Smart Contracts. The idea of connecting the real building to its digital model on the Blockchain platform redefines the paradigm of the BIM process. The use of Smart Contracts represents a potential solution to save time and costs during the execution of each phase. Nowadays, the adoption of Blockchain shows more potential in the design phase, where the use of BIM is already at its highest level. The combination of the two technologies offers enormous value and can be considered the appropriate direction for the efficient development of the sector (Penzes 2018). Even though it is possible to recognize many advantages, due to the novelty of the technology, the majority of operators don't have adequate training in its use and methods and, because parties are used to manage their information and activities through their register, the transition to a distributed system is difficult. For these reasons and the absence of a common regulation the researches, the trials and the adoptions of Blockchain in construction are still in the explanatory phase. Anyway, there is no doubt that the transition from hierarchical to distributed system promoted by DLTs adequately supports the development of the widespread nature of the built environment. Only through applications and case studies will be possible to understand the real impact and consequences of Blockchain application. Considering the outputs of the research carried out, it is possible to identify the most likely and feasible future research 
areas of Blockchain applications in (i) decision-making process, (ii) procurement process and (iii) payments release.

\section{References}

ARUP, Blockchain and the Built Environment, 2019.

Dounas, T., Lombardi, D., and Jabi, W., Towards Blockchains for Architectural Design Consensus Mechanisms for Collaboration in BIM, In Architecture in the Age of the 4th Industrial Revolution Proceedings of the 37th eCAADe and 23rd SIGraDi Conference, 2019.

Erri Pradeep, A. S., Yiu, T. W., and Amor, R., Leveraging Blockchain Technology in a BIM Workflow: A Literature Review, International Conference on Smart Infrastructure and Construction 2019 (ICSIC) 371-380, January, 2019.

European Commission, European Construction Sector Observatory Building Information Modelling in the EU construction sector, March 22, 2019.

European Construction Sector Observatory, European Construction Sector Observatory - Country Profile Italy, June 27, 2018.

Gálvez-Martos, J. L., Styles, D., Schoenberger, H., and Zeschmar-Lahl, B., Construction and Demolition Waste Best Management Practice in Europe, Resources, Conservation and Recycling, 136, 166-178, May, 2018.

Hsiao, J. I.-H., "Smart" Contract on the Blockchain-Paradigm Shift for Contract Law?, US-China Law Review, 14(10), 685-694, 2017.

Hunhevicz, J. J., and Hall, D. M., Managing Mistrust in Construction Using DLT: A Review of Use-Case Categories for Technical Decisions, Proceedings of the 2019 European Conference for Computing in Construction, 1, 100-109, 2019.

Koutsogiannis, A., and Berntsen, N., Blockchain and Construction: The How, Why and When, Chartered Institute of Building, 2017.

Lai, R., and Lee Kuo Chuen, D., Blockchain-From Public to Private 1st ed., Vol. 2, Elsevier Inc., 2017.

Li, J., Greenwood, D., and Kassem, M., Blockchain in The Built Environment and Construction Industry: A Systematic Review, Conceptual Models and Practical Use Cases, Automation in Construction, 102, 288307, January, 2019.

Mathews, M., Robles, D., and Bowe, B., BIM+Blockchain: A Solution to the Trust Problem in Collaboration?, CITA BIM Gathering 2017, 11, 2017.

McKinsey Global Institute, Reinventing Construction: A Route to Higher Productivity, McKinsey and Company, Feb 20, 2017.

Nawari, N. O., and Ravindran, S., Blockchain Technologies in BIM Workflow Environment, 593-602, 2019.

Osservatorio Blockchain and Distributed Ledger, Blockchain and Distributed Ledger: Unlocking The Potential of the Internet of Value, 2020.

Penzes, B., Blockchain Technology in The Construction Industry, Institution of Civil Engineers, 2018.

Rodrigues, B., Bocek, T., and Stiller, B., The Use of Blockchains: Application-Driven Analysis of Applicability 1st ed., Elsevier Inc., 111, 2018.

San, K. M., Choy, C. F., and Fung, W. P., The Potentials and Impacts of Blockchain Technology in Construction Industry: A Literature Review, IOP Conference Series: Materials Science and Engineering, 495(1), 012005, 2019.

Tezel, A., Papadonikolaki, E., and Yitmen, I., Preparing Construction Supply Chains for Blockchain: An Exploratory Analysis, CIB World Building Congress, June 17-21, 2019.

Turk, Z.., and Klinc, R., Potentials of Blockchain Technology for Construction Management, Procedia Engineering, 196, 638-645, June, 2017.

Ye, Z., Yin, M., Tang, L., and Jiang, H., Cup-of-Water theory: A Review on The Interaction of BIM, IoT and Blockchain During The Whole Building Lifecycle, ISARC 2018 - 35th International Symposium on Automation and Robotics in Construction and International AEC/FM Hackathon: The Future of Building Things (ISARC), 2018. 